\title{
Statin Attenuates Experimental Anti-Glomerular Basement Membrane Glomerulonephritis Together with the Augmentation of Alternatively Activated Macrophages
}

\author{
Emiko Fujita, ${ }^{\star \dagger}$ Akira Shimizu, ${ }^{*}$ Yukinari Masuda, ${ }^{*}$ \\ Naomi Kuwahara, ${ }^{*}$ Takashi Arai, ${ }^{*}$ \\ Shinya Nagasaka, ${ }^{*}$ Kaoru Aki, ${ }^{*}$ Akiko Mii, ${ }^{\dagger}$ \\ Yasuhiro Natori, ${ }^{\neq}$Yasuhiko lino, ${ }^{\dagger}$ \\ Yasuo Katayama, ${ }^{\dagger}$ and Yuh Fukuda* \\ From the Department of Analytic Human Pathology,* and the \\ Division of Neurology, Nephrology and Rheumatology, ${ }^{\dagger}$ \\ Department of Internal Medicine, Nippon Medical School, Tokyo; \\ and the Department of Health Chemistry, ${ }^{\ddagger}$ School of Pharmacy, \\ Iwate Medical University, Iwate, Japan
}

Macrophages are heterogeneous and include classically activated M1 and alternatively activated M2 macrophages, characterized by pro- and anti-inflammatory functions, respectively. Macrophages that express heme oxygenase- 1 also exhibit anti-inflammatory effects. We assessed the anti-inflammatory effects of statin in experimental anti-glomerular basement membrane glomerulonephritis and in vitro, focusing on the macrophage heterogeneity. Rats were induced anti-glomerular basement membrane glomerulonephritis and treated with atorvastatin $(20 \mathrm{mg} / \mathrm{kg} / \mathrm{day})$ or vehicle (control). Control rats showed infiltration of macrophages in the glomeruli at day 3 and developed crescentic glomerulonephritis by day 7 , together with increased mRNA levels of the M1 macrophage-associated cytokines, interferon- $\gamma$, tumor necrosis factor- $\alpha$, and interleukin-12. In contrast, statin reduced the level of proteinuria, reduced infiltration of macrophages in glomeruli with suppression of monocyte chemotactic protein-1 expression, and inhibited the formation of necrotizing and crescentic lesions. The number of glomerular ED3-positive macrophages decreased with down-regulation of M1 macrophage-associated cytokines. Furthermore, statin augmented ED2-positive M2 macrophages with up-regulation of the M2 macrophage-associated chemokines and cytokines, chemokine (C-C motif) ligand-17 and interleukin-10. Statin also increased the glomerular interleukin-10-expressing heme oxygenase-1-positive macrophages. Statin inhibited macrophage development, and suppressed ED3-positive macrophages, but augmented ED2-positive macrophages in M2-associated cytokine environment in vitro. We conclude that the anti-inflammatory effects of statin in glomerulonephritis are mediated through inhibition of macrophage infiltration as well as augmentation of anti-inflammatory macrophages. (Am J Pathol 2010, 177:1143-1154; DOI: 10.2353/ajpath.2010.090608)

Statins, inhibitors of 3-hydroxy-3-methylglutaryl CoA reductase, are widely used for the treatment of hyperlipidemia through reduction of cholesterol synthesis. In addition to their lipid lowering effect, recent evidence suggests that statins have other important properties, such as anti-atherogenic and tissue-protective functions. These functions of statin are mediated by blocking 3-hydroxy-3-methylglutaryl CoA reductase, and thereby inhibition of cholesterol synthesis, as well as blockade of the mevalonate pathway and the synthesis of isoprenoids (farnesyl pyrophosphate and geranylgeranyl pyrophosphate). Isoprenoids are essential for the post-translational modification of several proteins involved in important signaling pathways, and their inhibition would interfere with numerous important cellular functions, thus adding many additional "pleiotropic" effects for statins, such as protection of endothelial function, antioxidant effects, antithrombotic effects, and anti-inflammatory and immunomodulatory functions. ${ }^{1,2}$ Other studies have also demonstrated that statins have beneficial effects in renal diseases and impede the progression of renal injury through their anti-inflammatory and immunomodulatory effects. ${ }^{3}$

Accepted for publication May 26, 2010

None of the authors disclosed any relevant financial relationships.

Address reprint requests to Akira Shimizu, M.D., Ph.D., Department of Analytic Human Pathology, Nippon Medical School, 1-1-5, Sendagi, Bunkyo-ku, Tokyo 113-8602, Japan. E-mail: ashimizu@nms.ac.jp. 
In various kidney diseases, infiltrating macrophages are found in renal inflammatory sites, and their presence in the interstitium and glomeruli contributes to the severity of tissue injury and progression of renal diseases. ${ }^{4-9}$ Indeed, interstitial macrophages are involved in the extension of interstitial inflammation, microvascular injury, and progression of interstitial fibrosis. ${ }^{5-12}$ In addition, macrophages infiltrating the glomeruli enhance glomerular injury in various forms of glomerulonephritis (GN), such as antineutrophil cytoplasmic antibodies-associated GN, anti-glomerular basement membrane (GBM) GN, lupus nephritis, cryoglobulinemia, mesangioproliferative GN, IgA nephropathy, and diabetic nephropathy. ${ }^{5,7,8,13,14}$ Furthermore, in experimental anti-GBM GN in Wistar-Kyoto (WKY) rats, activated macrophages are the direct cause of necrotizing and crescentic glomerular lesions. ${ }^{15-19}$

In the macrophage-associated inflammatory process, recent studies have focused on the heterogeneity of macrophage activation and in particular their ability to amplify or curtail inflammation. ${ }^{20}$ In response to environmental milieu, macrophage changes can give rise to different populations of cells with distinct functions that are categorized as either classically activated (M1) or alternatively activated (M2). ${ }^{21}$ Classically activated $\mathrm{M} 1$ macrophages are tissue injury type macrophages involved mainly in the expansion of inflammation. ${ }^{13,22}$ On the other hand, the M2 macrophages have immunoregulatory and immunosuppressive functions. In addition, heme oxygenase-1 (HO-1) positive (+) macrophages act as cytoprotective anti-inflammatory macrophages by local delivery of $\mathrm{HO}-1 .{ }^{23}$ Thus, in addition to the numbers of infiltrating macrophages, macrophage phenotype is important in determining the outcome of inflammatory disease.

While statins decrease the total number of infiltrating macrophages in renal diseases,,$^{3,24-26}$ it remains uncertain whether statins affect the composition of the macrophage population. In the present study, we investigated the influence of statin on macrophage phenotype, particularly pro-inflammatory and anti-inflammatory macrophages, in a rat model of macrophage-mediated immune glomerular injury induced by injection of anti-GBM antibody in WKY rats.

\section{Materials and Methods}

\section{Induction of Anti-GBM GN in WKY Rats}

The Ethics Review Committee for Animal Experimentation of Nippon Medical School approved the animal experiments described in the present study. Inbred male WKY rats (Charles River Japan, Kanagawa, Japan) that weighed $100 \mathrm{~g}$ were used in this study. Anti-GBM GN was induced by injection of rabbit anti-rat GBM antibody at a dose of $50 \mu \mathrm{g} \mathrm{lgG} / 100 \mathrm{~g}$ body weight on day $0 .{ }^{15-17}$ The rats were either administered oral atorvastatin (20 $\mathrm{mg} / \mathrm{kg}$ body weight/day) (statin group) or vehicle (vehicle/control group) from 3 days before the injection of anti-GBM antibody through the day of sacrifice. We decided the dose of atorvastatin by the referring to the studies that demonstrate the anti-inflammatory effects of statin in several experimental models of inflammatory and autoimmune diseases as well as kidney transplantation. ${ }^{11,27,28}$ Atorvastatin was a generous gift from Pfizer (New York, NY). In each group, 10 rats underwent biopsy or were sacrificed at days $0,3,5$, or 7 . For estimating renal function, urine and blood samples were assayed for urinary protein and serum levels of total cholesterol, triglyceride, and high-density lipoprotein cholesterol using an autoanalyzer (SRL, Tokyo, Japan).

\section{Histopathology, Immunohistochemistry, and Electron Microscopy}

After removal of the kidney, renal tissues were fixed in $20 \%$ buffered formalin and embedded in paraffin for light microscopic examination. Tissues were stained with H\&E, periodic acid-Schiff, and periodic acid-silver methenamine for histopathological examination. The following primary antibodies were used for immunohistochemistry: 1) Monoclonal mouse anti-rat ED1 antibody (BMA, Nagoya, Japan) for detection of infiltrating macrophages. 2) Monoclonal mouse anti-proliferating cell nuclear antigen (PCNA) antibody (PC10; DAKO, Glostrup, Denmark), a marker for cellular proliferation. 3) Monoclonal mouse anti-rat $\mathrm{HO}-1$ and polyclonal rabbit anti-rat $\mathrm{HO}-1$ antibody (Stressgen, Victoria, BC, Canada) for detection of $\mathrm{HO}-1$ expressing cells. 4) Monoclonal mouse anti-rat ED2 antibody (BMA, Nagoya, Japan) for detection of M2 macrophages, because rat ED2 is CD163, which is expressed on M2 macrophages. ${ }^{22,29,30}$ 5) Monoclonal mouse anti-rat ED3 antibody (BMA) for detection of activated macrophages. Rat ED3 is known for tissue fixed macrophages, though bone marrow-derived macrophages stimulated by $T$ cells are positive for ED3, indicating that anti-rat ED3 antibody can detect activated

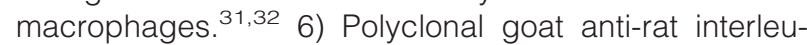
kin-10 (IL-10) antibody (R\&D Systems, Minneapolis, MN) for detection of IL-10-expressing cells. 7) Polyclonal goat anti-monocyte chemotactic protein-1 (MCP-1) antibody (Santa Cruz Biotechnology), 8) polyclonal goat anti-tumor necrosis factor- $\alpha$ (TNF- $\alpha$ ) antibody (Santa Cruz Biotechnology), and 9) polyclonal goat anti-inducible nitric oxide synthesis (iNOS) antibody (Santa Cruz Biotechnology) for detection of cells that produce or express M1 macrophage-related chemokine, cytokine, or enzyme. 10) Polyclonal goat anti-CC chemokine ligand 17 (CCL17) antibody (Santa Cruz Biotechnology) for detection of M2-related chemokine-expressing cells.

For immunohistochemistry for ED1 and PCNA, 20\%buffered, formalin-fixed, paraffin-embedded tissue sections were used and the specimens were stained by the standard avidin-biotin-peroxidase complex technique. For ED1 and PCNA, tissue sections were incubated with $0.1 \%$ pepsin for 45 minutes ( $\mathrm{min}$ ) and microwaved for 10 minutes in $0.01 \mathrm{~mol} / \mathrm{L}$ sodium citrate $(\mathrm{pH} \mathrm{6.0)}$, respectively, before incubation with the primary antibody. To evaluate the proliferating macrophages, double immunohistochemistry with PCNA and ED1 was performed using the color modification method of 3,3'-diaminobenzidine 
precipitation by nickel chloride, which changes diaminobenzidine color from brown to black. ${ }^{15,17,33}$ Sections were incubated with biotinylated anti-rat ED1 antibody and an avidin-biotin peroxidase complex followed by $\mathrm{H}_{2} \mathrm{O}_{2}$ containing diaminobenzidine. Sections were then incubated with PCNA followed by peroxidase-conjugated goat anti-mouse lgG, and $\mathrm{H}_{2} \mathrm{O}_{2}$ and nickel chloride-containing diaminobenzidine. For detection of ED2, ED3, HO-1, MCP-1, TNF- $\alpha$, iNOS, CCL17, and IL-10+ cells in glomeruli, $4-\mu \mathrm{m}$ frozen sections were stained by the standard indirect technique and observed under a confocal scanning laser microscopy (TCS-SP5, Leica Lasertechnic, Heidelberg, Germany) based on an upright microscope (DM6000B, Leica Lasertechnic) equipped with a krypton-argon laser. For detecting $\mathrm{HO}-1$ expression on ED1+ macrophages and ED2+ macrophages, double immunohistochemical staining with $\mathrm{HO}-1$ (mouse $\operatorname{lgG}_{2 \mathrm{~b}}$; fluorescein isothiocyanate [FITC]) and ED1 (mouse $\operatorname{lgG}_{1}$; Texas-red) or ED2 (mouse IgG 1 ; Texas-red) was performed. For detection of IL-10 production by ED2+ and HO-1+ macrophages, double immunohistochemical staining with IL-10 (goat IgG; Texas-red) and ED2 (mouse $\operatorname{lgG}_{1}$; FITC) or HO-1 (mouse $\operatorname{lgG}_{1}$; FITC) was performed. Both ED2+ and ED3+ cells that may have both characterization of $\mathrm{M} 1$ and $\mathrm{M} 2$ were examined by double immunohistochemical staining for ED2 (mouse $\operatorname{lgG}_{1}$; Texas Red) and ED3 (mouse $\operatorname{lgG}_{2 a}$; FITC). In addition, MCP- 1 , TNF- $\alpha$, iNOS, and CCL17 expressing cells were examined by double immunohistochemical staining for MCP-1, TNF $\alpha$, iNOS, as well as CCL17 (goat IgG) and ED3 or ED2 (mouse IgG). For indirect immunofluorescence, nonspecific binding sites were blocked with 10\% normal rat serum (Rockland Immunochemicals Inc., Gilbertsville, PA) for 30 minutes. For all biopsies, negative controls were used in which the primary antibody was substituted with equivalent concentrations of an irrelevant antibody.

For electron microscopic examination, the kidney sections were fixed in $2.5 \%$ glutaraldehyde solution in phosphate buffer $(\mathrm{pH} 7.4)$ and post-fixed with $1 \%$ osmium tetroxide, dehydrated, and embedded in Epok 812. UItrathin sections were stained with uranyl acetate and lead citrate and then examined with an electron microscope (model H7100, Hitachi Corp., Tokyo, Japan).

\section{Real-Time PCR Using Isolated Glomeruli}

To examine the mRNA levels of IFN- $\gamma$, TNF- $\alpha$, IL-12, IL-4, MCP-1, CCL17, mannose receptor, HO-1, and IL-10, realtime PCR was performed using isolated glomeruli that were prepared by the standard three-stage sieving method. ${ }^{17,33}$ The glomerular total RNA was extracted from isolated glomeruli by Qiagen RNeasy Mini kit (Qiagen, Hilden, Germany). A cDNA copy was created with reverse transcriptase from High Capacity RT-kit (Applied Biosystems, Foster City, CA). Gene expression was analyzed by real-time quantitative RT-PCR using the TaqMan system based on real-time detection of accumulated fluorescence according to the manual supplied by the manufacturer (ABI PRISM 7900HT; Applied Biosystems). The normalized value for
mRNA expression in each sample was calculated as the relative quantity of relevant primers divided by the relative quantity of the housekeeping gene $18 \mathrm{~S}$ ribosomal RNA (Taqman Ribosomal RNA Control kit; Applied Biosystems). The sequences of the TaqMan sets were as follows: HO-1 (Rn00561387_m1), IFN- $\gamma$ (Rn00594078_ m1), MCP-1 (Rn00580555_m1), IL-4 (Rn01456866_m1), TNF- $\alpha$ (Rn99999017_m1), IL-10 (Rn00563409_m1), mannose receptor (Rn01456616_m1), and IL-12 (Rn00575112 m1). Quantification was performed using SDS 2.1 software.

\section{Circulating Monocytes in Vivo and Macrophage Activation in Vitro}

To examine the effects of statin on the circulating monocytes/macrophages after the induction of anti-GBM GN the number of circulating monocytes/macrophages and characterization of macrophages (ED1, ED2, and ED3) were assessed by the flow cytometric analysis in normal rats, rats in control group, and rats in statin group after the induction of anti-GBM GN.

The human monocytic cell line U937 (Cell No. JCRB9021) was obtained from Health Science Research Resources Bank (Osaka, Japan). Rat peritoneal macrophages (peritoneal resident cells) were collected from normal WKY rat by washing peritoneal cavities with the 20 $\mathrm{ml}$ ice cold RPMI 1640. U937 cells and rat peritoneal macrophages were maintained in RPMI1640 medium, supplemented with $10 \%$ fetal bovine serum, $50 \mu \mathrm{mol} / \mathrm{L}$ 2-mercaptoethanol, and antibiotics $(100 \mathrm{U} / \mathrm{ml}$ penicillin, and $100 \mu \mathrm{g} / \mathrm{ml}$ streptomycin) at $37^{\circ} \mathrm{C}$ in $5 \% \mathrm{CO}_{2}$. To detect the effects of statin on macrophage differentiation from U937, $5 \times 10^{5}$ U937 cells $/ \mathrm{ml}$ were incubated with phorbol 12-myristate 13-acetate (100 ng/ml; Sigma, St Louis, MO) and atorvastatin (0, 0.5, or $2.5 \mu \mathrm{mol} / \mathrm{L})$ for 3 days. Rat peritoneal macrophages were prepared as adherent cells 1 hour after they were incubated. To clarify the effects of statin on M1 or M2 differentiation from rat peritoneal macrophages, $5 \times 10^{5} \mathrm{cells} / \mathrm{ml}$ were incubated with lipopolysaccharide (LPS, Escherichia coli; Sigma; $1 \mu \mathrm{g} / \mathrm{ml}$ ) (M1 macrophage condition) or $10 \mathrm{ng} / \mathrm{ml}$ of recombinant rat IL-4 and human IL-13 (M2 macrophage condition) for 24 hours in the presence or in the absence of $2.5 \mu \mathrm{mol} / \mathrm{L}$ atorvastatin (statin started 1 day earlier than LPS or IL-4 and IL-13 stimulation). Cultured U937 cells were stained with FITC-conjugated anti-CD11b antibody (Beckman Coulter, Fullerton, CA) and allophycocyaninor phycoerythrin-conjugated anti-CD163 antibody (eBioscience), anti-mannose receptor antibody (BD Bioscience, San Jose, CA) or anti-CD169 antibody (Abcam, Cambridge, UK). For intracellular $\mathrm{HO}-1$ staining, cells were fixed with $4 \%$ paraformaldehyde and permeabilized with $0.5 \%$ Triton X. After blocking with PBS containing 3\% bovine serum albumin, cells were stained with rabbit anti-HO-1 antibody (Stressgen) following secondary staining with phycoerythrin-conjugated anti-rabbit IgG antibody (Santa Cruz Biotechnology). Cultured rat peritoneal macrophages were stained with mouse anti-ED2 antibody (AbD Serotec) or mouse anti-ED3 antibody (AbD Serotec) following the secondary staining with 
Table 1. Serum Lipid Profile in the Control (Vehicle) and Statin Groups

\begin{tabular}{lcccc}
\hline & \multicolumn{2}{c}{ Vehicle } & & Statin \\
\cline { 2 - 4 } & Day 0 & Day 7 & Day 0 & Day 7 \\
\hline T-Cho $(\mathrm{mg} / \mathrm{dL})$ & $118.8 \pm 5.0$ & $125.3 \pm 3.6$ & $119.5 \pm 7.9$ & $135.0 \pm 22.9$ \\
TG $(\mathrm{mg} / \mathrm{dL})$ & $58.0 \pm 19.2$ & $75.3 \pm 11.2$ & $51.3 \pm 21.0$ & $62.5 \pm 29.3$ \\
HDL-C $(\mathrm{mg} / \mathrm{dL})$ & $30.0 \pm 6.7$ & $35.5 \pm 5.3$ & $28.0 \pm 3.6$ & $31.3 \pm 5.2$ \\
\hline
\end{tabular}

T-Cho, total cholesterol; TG, triglyceride; HDL-C, high-density lipoprotein cholesterol.

FITC-conjugated rat anti-mouse IgG1 antibody (Biolegend) or rat anti-mouse IgG2a antibody (Biolegend). For intracellular ED1 staining, cells were fixed with $4 \%$ paraformaldehyde and permeabilized with $0.5 \%$ Triton X. After blocking them using PBS containing 3\% bovine serum albumin, cells were stained with phycoerythrin-conjugated anti-ED1 antibody (AbD Serotec). Flow cytometric analysis was performed on FACSCanto II and FACSDiva 6.1 software (Becton Dickinson, San Jose, CA). Eight-well chamber slides were observed under a confocal scanning laser microscopy (TCS-SP5, Leica Lasertechnic) based on an upright microscope (DM6000B, Leica Lasertechnic) equipped with a krypton-argon laser.

\section{Quantification of Histopathological Changes}

In each kidney sample, more than 30 cross-sections of glomeruli were examined sequentially for the following parameters: 1) proliferating macrophages: the mean number of both PCNA+ and ED1+ cells per glomerular cross section; 2) infiltrating macrophages: the mean number of ED1+ cells per glomerular cross section; 3 ) infiltrating ED2+ or ED3+ cells: the mean number of ED2+ or ED3+ cells per glomerular cross section; 4) infiltrating $\mathrm{HO}-1+$ cells: the mean number of $\mathrm{HO}-1+$ cells per glomerular cross section; 5) single ED3+, both ED3+ and ED2+, and single ED2+ cells, as well as MCP-1+, TNF- $\alpha+$, iNOS + , and CCL-17+ cells in ED3+ cells or ED2 + cells: the mean percentage of positive cells in glomeruli in double immunostain with ED3 and ED2, or in double immunostain with MCP-1, TNF- $\alpha$, iNOS, or CCL-17 cells and ED3 or ED2; 6) necrotizing and crescentic glomeruli: the mean percentage of glomeruli with necrotic and crescentic lesions in periodic acid-silver methenamine-stained sections. Glomerular cross-sections that contained only a small portion of the glomerular tuft were excluded from the analysis. All histopathological evaluations were performed by investigators who were blinded to the clinical information.

The data were expressed as the mean \pm SD. Statistical analysis was performed using Mann-Whitney's Utest. A $P$ value $<0.05$ denoted the presence of statistical significance.

\section{Results}

\section{Serum Lipid Profiles}

The levels of total cholesterol, triglyceride, and highdensity lipoprotein cholesterol were not significantly different between before and after statin administration as well between the statin and control groups throughout the experiment (Table 1).

\section{Inflammatory Cell Infiltration and Crescentic Glomerular Lesions in Anti-GBM GN}

A single injection of anti-rat GBM antibody on day 0 resulted in severe necrotizing and crescentic GN in WKY rats. In the control group, accumulation of glomerular inflammatory cells was noted at day 3 , which was followed by severe necrotizing and crescentic lesions at days 5 to 7 (Figure 1). In contrast, in the statin group, mild mononuclear cells infiltrated the glomeruli at day 7 with minimal necrotizing and crescentic lesions (Figure 1 and Figure 2A). A significant reduction of urinary protein excretion was observed in the statin group compared with

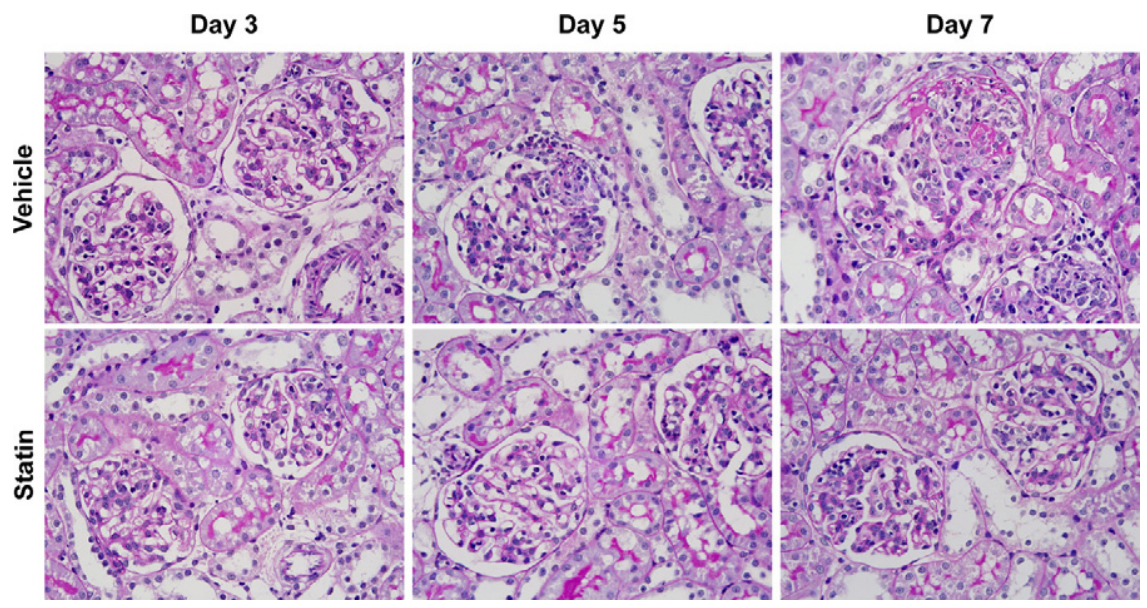

Figure 1. Representative photomicrographs showing histopathological changes in anti-GBM GN in the control (vehicle) and statin groups (periodic acid-Schiff stain, $\times 400$ ). In the control group, glomerular inflammatory cells accumulated at day three, and necrotizing and crescentic lesions developed by day seven. In contrast, statin reduced the number of inflammatory cells in glomeruli and inhibited the frequency of necrotizing and crescentic lesions at day seven. 


\section{A Necrotizing and crescentic glomeruli}

(\%)

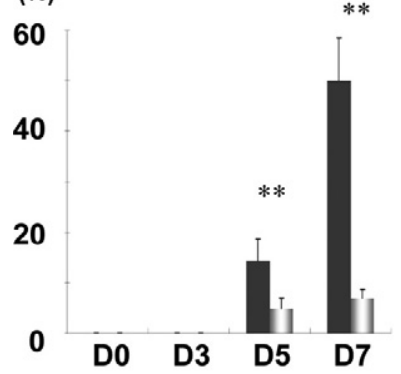

B

Proteinuria (mg/day)

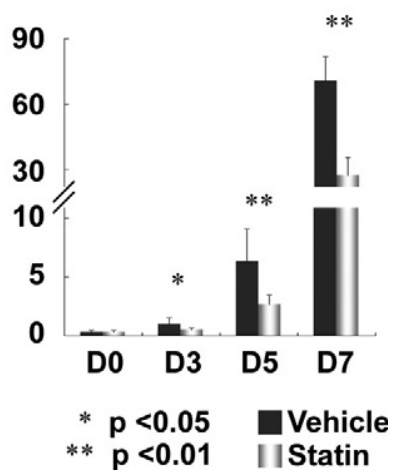

Figure 2. Percentage of necrotizing and crescentic glomeruli (A) and urinary protein excretion (B) in the control (vehicle) and statin groups. Statin administration decreased significantly proteinuria and necrotizing and crescentic glomerular lesions by day seven. Data are mean $\pm \mathrm{SD} ; n=5$ in each time point in each group.

that the control group at days 3 to 7 (Figure 2B). Despite the severe renal damage, serum creatinine levels did not increase at day 7 after induction of anti-GBM GN even in the control group, and no significant differences in serum creatinine levels were evident between the statin and control groups (data not shown).

\section{Effects of Statin on Macrophage Accumulation, Proliferation, and Activation}

After induction of anti-GBM GN, many infiltrating ED1+ macrophages were noted in the glomeruli at days 5 to 7 in the control group (Figure 3). By contrast, statin reduced ED1+ macrophage infiltration in glomeruli at day 7. Administration of statin decreased significantly the number of infiltrated ED1+ cells at days 3 and 7, compared with those in the control group (Figure 4A). Using PCNA expression as a marker of cell proliferation, many ED1+ PCNA + proliferating macrophages were noted in the glomeruli of the control group. However, statin inhibited significantly the proliferation of macrophages

in glomeruli at day 5 and 7 (Figure 4B and Figure 5). In addition, statin suppressed macrophage activation (enlargement in size and villi formation) and inhibited the infiltration into glomerular subendothelial spaces and mesangial areas from capillary lumens at day 7 (Figure 5). Statin also suppressed the up-regulation of mRNA levels of glomerular MCP-1 after disease induction at days 3 to 7 (Figure 4C). These findings suggested that statin did not only suppress macrophage infiltration into glomeruli through low expression of MCP-1, but also inhibited macrophage activation and proliferation at inflammatory sites.

\section{Statin Reduces Activated Macrophages and Inhibits the Production of IFN- $\gamma$, TNF- $\alpha$, and IL-12 in Glomeruli}

In the present study, we examined activated macrophages using rat ED3. ${ }^{31,32}$ Many ED3+ macrophages were observed in glomeruli of the control group (Figure $6 A)$. These ED3 + cells included many ED3 single positive cells and a few of both ED3 + and ED2 + cells and a few of ED2 single positive cells (Figure 7). In the statin group, only a few ED3+ cells infiltrated the glomeruli (Figure 6B). Statin significantly reduced the number of glomerular ED3+ macrophages at days 3 and 7 (Figure 6C). Glomerular infiltrating ED3+ macrophages expressed MCP-1, TNF- $\alpha$, and iNOS (Figure 7). In real-time PCR of isolated glomeruli, overexpression of pro-inflammatory cytokines, including IFN- $\gamma$, TNF- $\alpha$, and IL-12, was noted in the control group, but such upregulation was significantly inhibited at day 7 following statin administration (Figure 6, D-F). Activation of M1 macrophages by classical immune pathways involves an IFN- $\gamma$-dependent Th1-type response, and exposure to IFN- $\gamma$ and TNF- $\alpha$ induces M1 polarization characterized by production of $\mathrm{IL}-12 .^{21}$ These findings indicated the enhancement of M1 polarization in anti-GBM antibodyinduced glomerular injury, which was inhibited by statin administration.
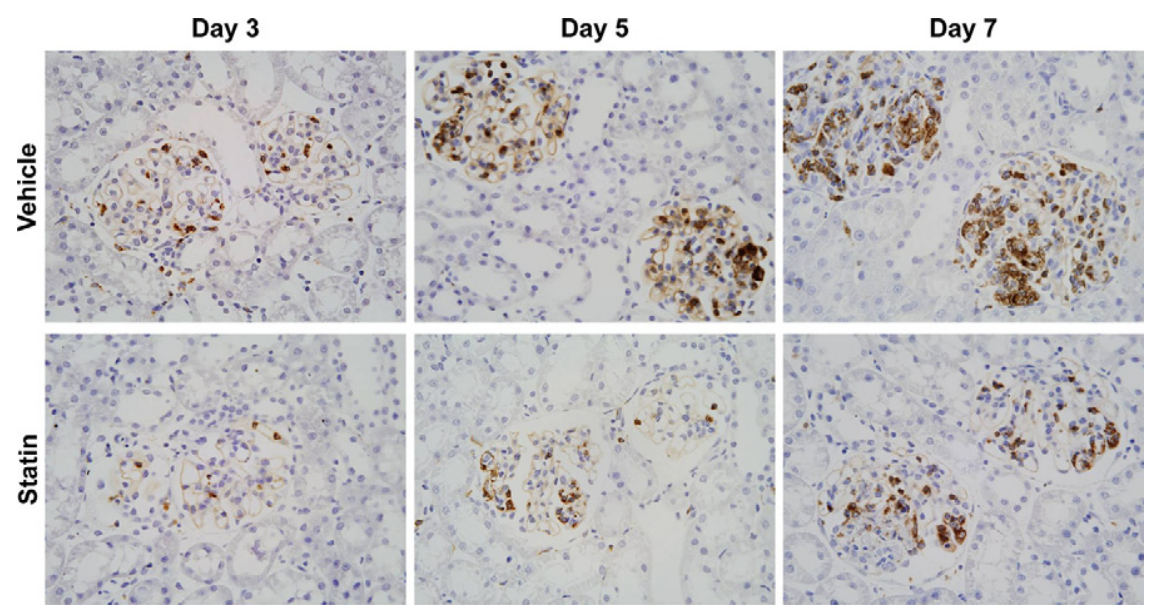

Figure 3. Representative photomicrographs showing ED1-positive macrophages in glomeruli of the control (vehicle) and statin groups (ED1 stain, $\times 400$ ). Many ED1-positive macrophages infiltrated the glomeruli at days five to seven in the control group. In contrast, statin reduced the number of glomerular ED1-positive macrophages throughout the experiment. 


\section{A ED1+ macrophages}

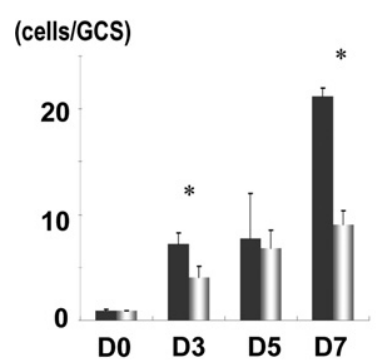

\section{B ED1+/PCNA+ cells}

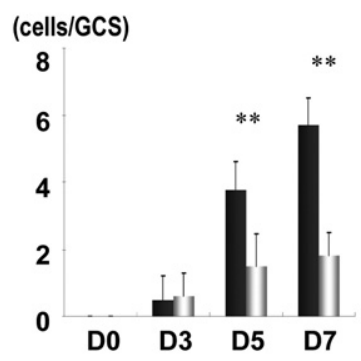

\section{C mRNA (normalized to 18S) \\ 40 \\ MCP-1}

30

20

10
Figure 4. Numbers of ED1-positive macrophages (A) and of ED1- and PCNA-positive proliferating macrophages (B) per glomerular cross section (GCS). mRNA levels of MCP-1 (C) in isolated glomeruli of control (vehicle) and statin groups. Statin reduced infiltrating ED1-positive macrophages and proliferating ED1 and PCNApositive macrophages in glomeruli by day seven. In addition, statin suppressed the upregulation of mRNA levels of MCP-1 in glomeruli at days five to seven. Data are mean $\pm \mathrm{SD} ; n=$ 5 in each time point in each group.

\section{Statin Augments Anti-Inflammatory Macrophages and Increases CCL17 and IL-10 Production in Glomeruli}

Rat ED2 + macrophages are considered as a subset of "alternatively activated" macrophages, because ED2 is identified CD163, which is known to be expressed on alternatively activated M2 macrophages. ${ }^{22,29,30}$ A few $\mathrm{ED} 2+$ macrophages were observed in the glomeruli of the control group. By contrast, many ED2+ macrophages were present in the glomeruli of the statin group (Figure 8, A and B). Quantitative analysis confirmed that statin increased significantly the number of glomerular ED2+ macrophages in a time-dependent manner (Figure 8C). M2 macrophages are mediated by various stimuli, including IL-4, and preferentially express receptors for foreign antigens, such as mannose receptor. ${ }^{13,20-22,30}$ In addition, M2 macrophages can produce CCL17. ${ }^{30,34,35}$ Glomerular infiltrating ED2+ macrophages expressed CCL17 (Figure 7). Real-time PCR of isolated glomeruli showed that statin up-regulated significantly the mRNA levels of IL-4, mannose receptor, and CCL17, compared with the control group (Figure 8, D-F). These findings indicated that statin stimulated the development of antiinflammatory M2 macrophages and augmented CCL17 expression in glomerular inflammation.

We also examined the involvement of $\mathrm{HO}-1+$ macrophages in the suppression of anti-GBM GN. In double immunostaining for $\mathrm{HO}-1$ and ED1, many $\mathrm{HO}-1+$ cells also expressed ED1+, indicating that ED1+ macrophages are the major source of $\mathrm{HO}-1+$ cells in the glomeruli (Figure 9, A and B). Administration of statin increased significantly the number of $\mathrm{HO}-1+$ cells in glomeruli at days 5 and 7 (Figure 9C), and up-regulated HO-1 mRNA levels in isolated glomeruli at days 3 to 7 , compared with the control group (Figure 9F). Importantly, double immunostaining for $\mathrm{HO}-1$ and ED2 indicated that many $\mathrm{HO}-1+$ macrophages also expressed ED2 in glomeruli (Figure 9, D and E).

Finally, we examined IL-10-expressing cells. In double immunostaining for IL-10 and ED2 in the statin group, ED2+ macrophages expressed IL-10 (Figure 10, A and B). Interestingly, double immunostaining for $\mathrm{IL}-10$ and $\mathrm{HO}-1$ identified IL-10-expressing $\mathrm{HO}-1+$ macrophages in the statin group (Figure 10, D and E). Furthermore, the mRNA levels of IL-10 were up-regulated significantly in

\section{ED1+/PCNA+ cells}

Day 7
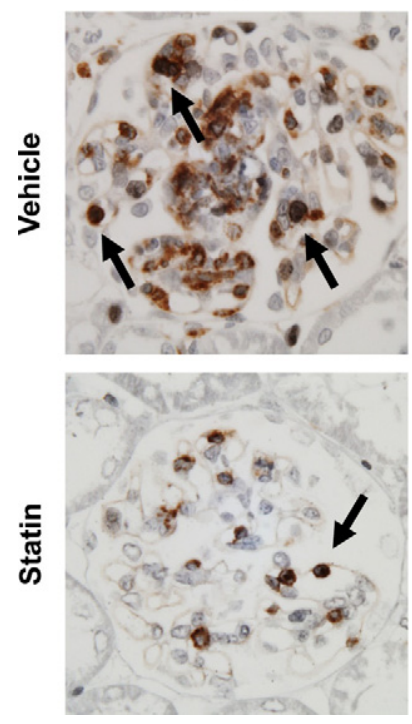

Day 5
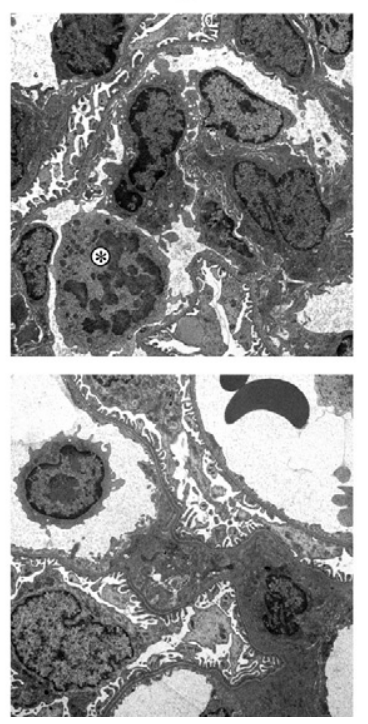

Day 7
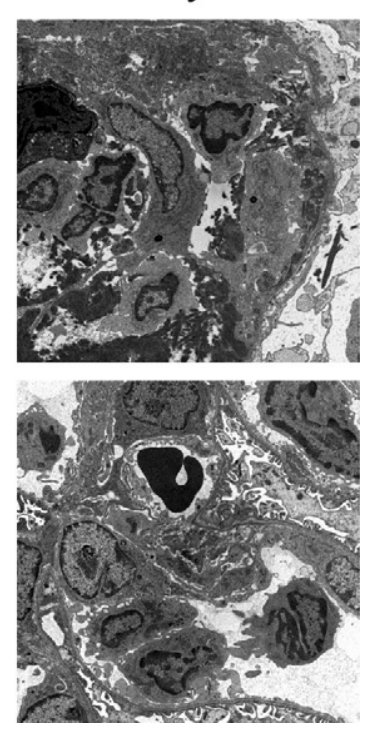

Figure 5. Representative photomicrographs showing proliferation and activation of macrophages in the control (vehicle) and statin groups (double immunohistochemistry for ED1 (brown) and PCNA (black). Arrows indicate both PCNAand ED1-positive proliferating macrophages, $\times 600$; electron microscopy $\times 6000)$. In the control group at day 7, many of the ED1-positive infiltrating macrophages were proliferating PCNA-positive macrophages in glomeruli. However, statin inhibited proliferation of macrophages in glomeruli. Electron microscopic findings at day five showed enlargement of glomerular macrophages in the control group with numerous villi that touched endothelial cells and other macrophages. The asterisk represents a macrophage in mitosis. At day seven, necrotizing lesions developed with loss of endothelial cells, accumulation of macrophages, and fibrin exudation in the control group. In contrast, macrophages were present mainly in the glomerular capillary lumen and did not infiltrate into the glomerular subendothelial space or mesangial areas in the statin group. 

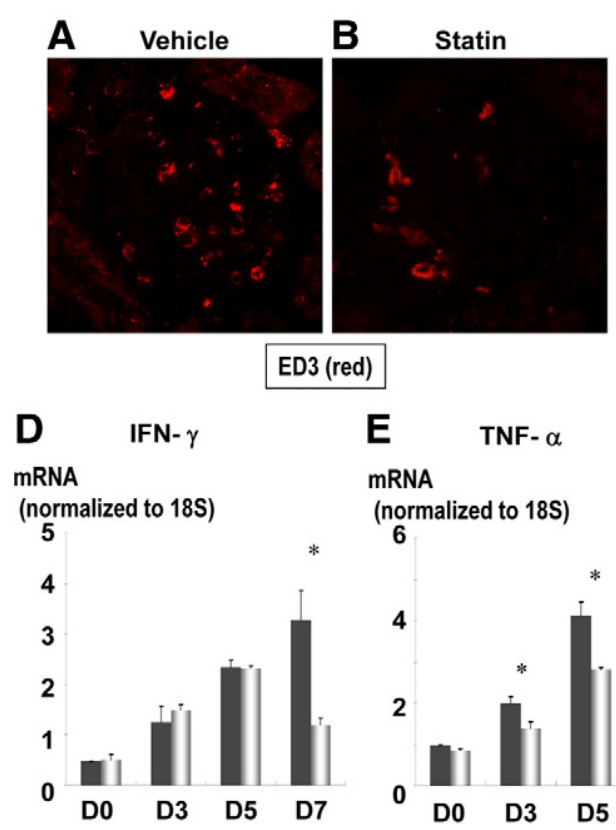

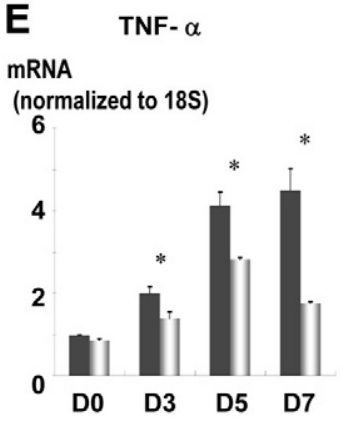

\section{The number of ED3+ cells (cells/GCS)}

15

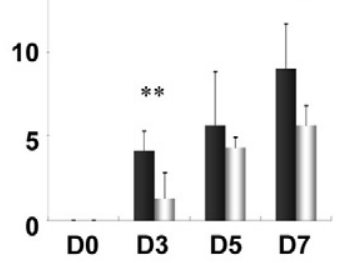

$\mathbf{F}$

IL-12

mRNA

(normalized to 18S)

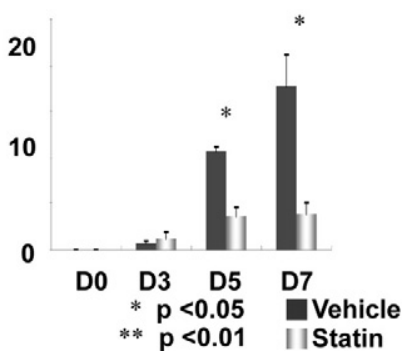

Figure 6. Activated ED3-positive macrophages and M1 macrophage-related cytokines in glomeruli of the control (vehicle) and statin groups. A, B: An immunofluorescence study of ED3positive activated macrophages at day seven (rat ED3; red) showed the presence of many ED3positive infiltrating macrophages in the glomeruli of the control group at day seven as compared with the statin group. C: Numbers of ED3positive cells per glomerular cross section (GCS) showed that statin significantly reduced the number of glomerular ED3-positive macrophages at days three and seven. The mRNA levels of (D) IFN- $\gamma,(\mathbf{E})$ TNF- $\alpha$, and (F) IL-12 in the control and statin groups showed that these M1 macrophage-related cytokines were up-regulated in the control group at days three to seven. However, in the statin group, mRNA levels of these cytokines were inhibited by day seven. Data in (C-F) are mean $\pm \mathrm{SD} ; n=5$ in each time point in each group. isolated glomeruli from the statin-treated rats at days 3 to 7, compared with the control group (Figure 10C). These results indicated that statin increased the production of IL-10, which was associated with $\mathrm{ED} 2+$ and $\mathrm{HO}-1+$ macrophages in glomerular inflammation.

\section{The Effects of Statin on}

\section{Monocytes/Macrophages in Vivo and Macrophage Activation in Vitro}

The number of circulating monocytes examined by the flow cytometric analysis did not change after the induction of anti-GBM GN with or without statin $(1.01 \pm 0.48 \times$ $10^{5} \mathrm{cells} / \mathrm{ml}$ in normal rats, $0.71 \pm 0.40 \times 10^{5} \mathrm{cells} / \mathrm{ml}$ in control rats, and $1.21 \pm 1.73 \times 10^{5}$ cells $/ \mathrm{ml}$ in statin group rats). In addition, ED2+ and ED3+ macrophages could not be detected in peripheral blood in normal rats, rats in control group, and rats in statin group on day 7 (data not shown).

To study the effects of statin on macrophage differentiation from monocytes, human monocytic cell line U937 was treated with phorbol 12-myristate 13-acetate in the absence or in the presence of statin for 3 days (Figure 11, $A$ and $B$ ). Statin inhibited CD11b expression (macrophage differentiation marker) in U937 cells in a dose dependent manner. In CD11b+ U937 cells after 3 days of culture, $\mathrm{HO}-1$ expression was significantly increased when U937 cells were treated with $2.5 \mu \mathrm{mol} / \mathrm{L}$ statin. The
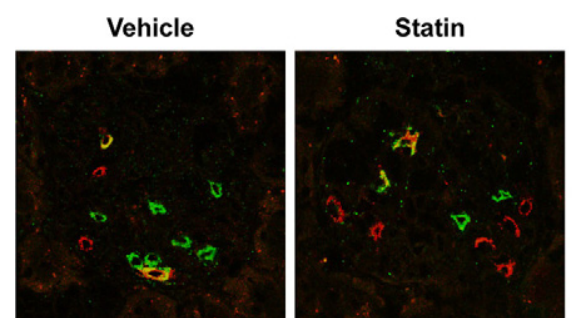

ED2 (red) ED3 (green)
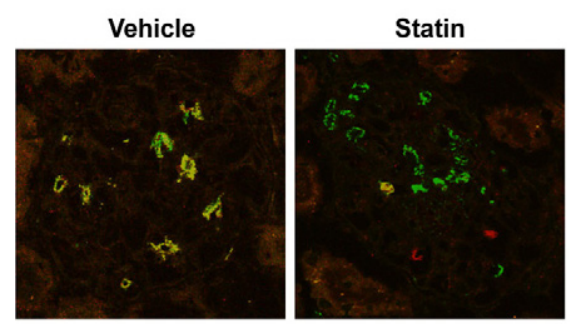

TNF- $\alpha$ (red) ED3 (green)
TNF- $\alpha$ (red) ED2 (green)

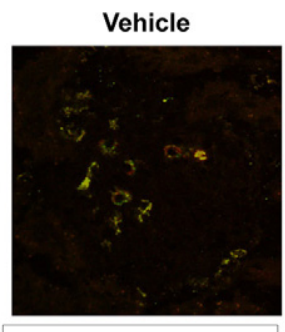

MCP-1 (red) ED3 (green)

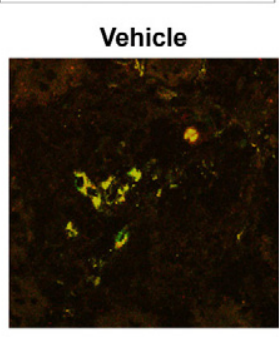

iNOS (red) ED3 (green)

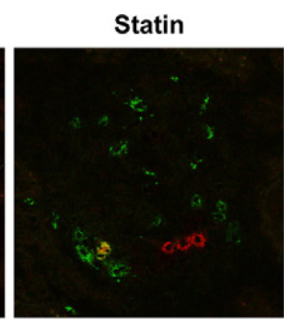

MCP-1 (red) ED2 (green) Statin

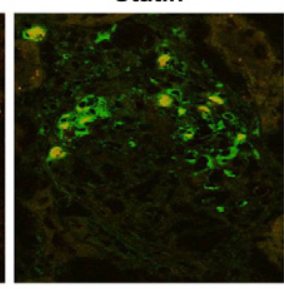

CCL17 (red) ED2 (green)
Figure 7. The characterization of glomerular infiltrating macrophages in the control (vehicle) and statin groups. In the control group at day seven, glomerular infiltrating ED3-positive cells were consisted of many ED3 single positive cells $(61.4 \pm 7.3 \%)$, a few of both ED3- and ED2positive cells $(18.8 \pm 4.9 \%)$ and a few ED2 single positive cells $(18.6 \pm 4.4 \%)$. By contrast, in the statin group at day seven, many ED2 single positive cells were evident in the glomeruli $58.8 \pm$ $13.4 \%$ ) with a few of both ED3-positive and ED2-positive cells $(23.2 \pm 9.2 \%)$ and a few of ED3 single-positive cells $(16.8 \pm 4.9 \%)$. Al MCP-1, TNF- $\alpha$, or iNOS producing cells were included in ED3-positive macrophages. MCP-1 and TNF- $\alpha$ were also expressed on a few ED2positive cells $(17.5 \pm 7.6 \%$ and $15.8 \pm 7.8 \%$ respectively), which were probably both ED2and ED3-positive cells, because single positive cells with MCP-1 and TNF- $\alpha$ could not be detected in double staining with ED3 and MCP-1 or TNF- $\alpha$. All CCL17 producing cells were included in ED2-positive macrophages. 

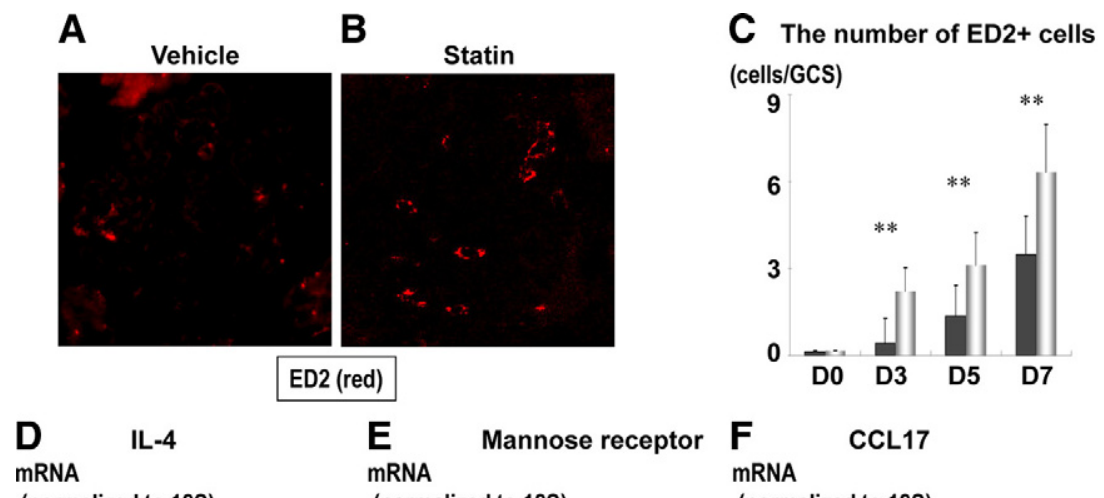

(normalized to 18S)

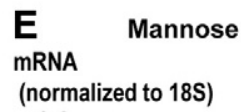

(normalized to 18S)

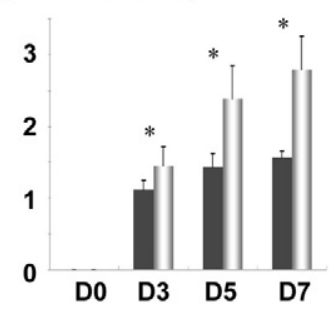

1.6

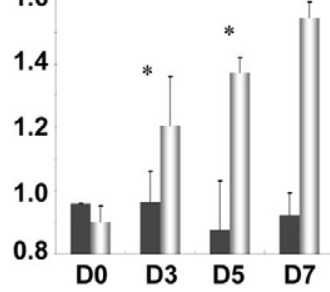

$\mathbf{F}$ mRNA

(normalized to 18S)

12

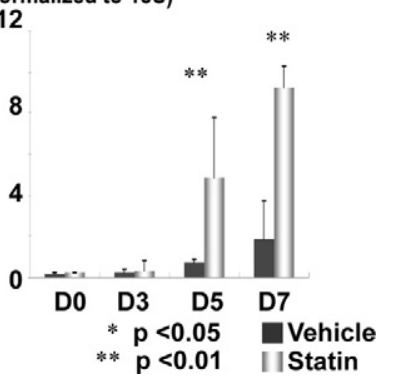

Figure 8. Anti-inflammatory M2 macrophages and related protein, chemokine, and cytokine in glomeruli of the control (vehicle) and statin groups. A, B: An immunofluorescence study of ED2-positive cells at day 7 (rat ED2; red) and (C) numbers of ED2-positive cells per glomerular cross section (GCS) showed the small number of ED2-positive cells in the control group. However, statin significantly increased the proportion of ED2-positive cells in the glomeruli. The mRNA levels of IL-4 (D; M2 macrophage-inducible cytokine), mannose receptor (E; M2 macrophage-related protein), and CCL17 (F; M2 macrophage-related chemokine) in the control and statin groups showed that these M2 macrophages and related protein, chemokine, and cytokine were up-regulated in the statin group when compared with those in the control group. Data in $\mathbf{C}-\mathbf{F}$ are mean $\pm \mathrm{SD} ; n=5$ in each time point in each group. expression of CD163, mannose receptor, and CD169 on U937 cells were not significantly different between absence and presence of statin (data not shown). To clarify the effects of statin on M1 or M2 differentiation, rat peritoneal macrophages were treated with LPS (M1 differentiation) or IL-4 and IL-13 (M2 differentiation) in the presence and in the absence of statin for 24 hours (statin started 1 day earlier than LPS or IL4 and IL-13 stimulation) (Figure 12). In the absence of statin, $46.9 \%$ of the
ED1+ cells expressed ED3 24 hours after LPS stimulation. However, there were $28.9 \%$ in the presence of statin. On the other hand, $21.7 \%$ of ED1+ cells expressed ED2 24 hours after IL-4 and IL-13 stimulation in the absence of statin, although there were $62.5 \%$ in the presence of statin. In addition, statin inhibited the morphological changes of macrophages that were characterized by spindle, stellate, polygonal shaped cells after LPS or IL-4 and IL-13 stimulation.
A

A Vehicle

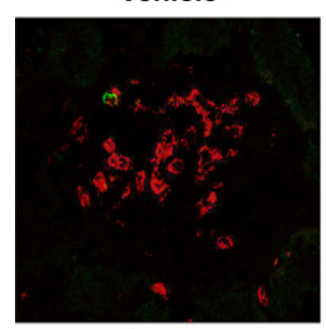

B

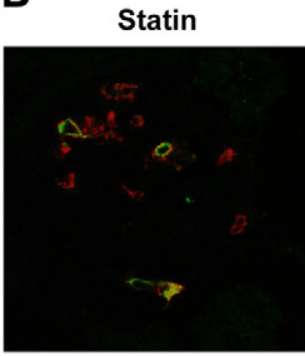

HO-1 (green) ED1 (red)

D

E
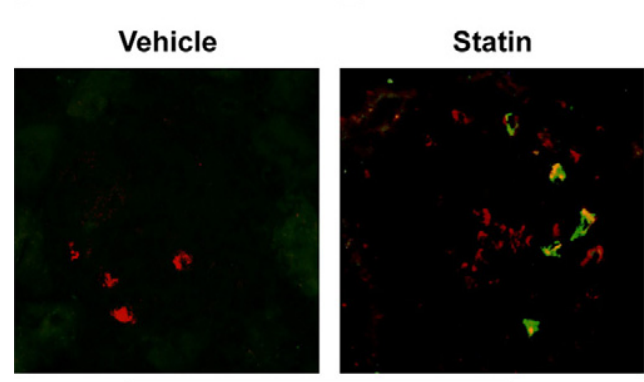

HO-1 (green) ED2 (red)
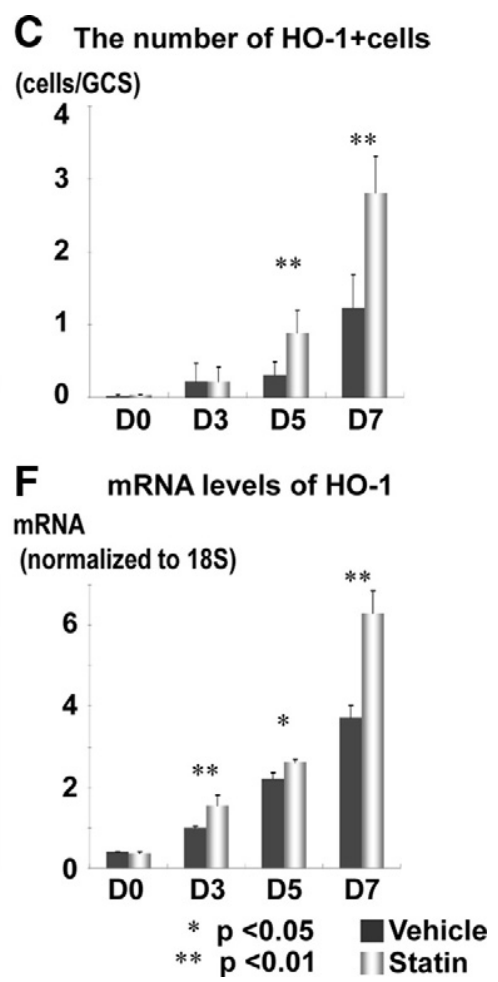

Figure 9. HO-1-positive macrophages in glomeruli in the control (vehicle) and statin groups. A, B: Double immunostaining for $\mathrm{HO}-1$ (green) and ED1 (red) showed both HO-1 and ED1positive macrophages in glomeruli of statintreated rats. C: Numbers of HO-1-positive cells per glomerular cross section (GCS) of the control and statin groups showed that statin significantly enhanced glomerular HO-1-positive macrophages in glomeruli at days 5 and 7 as compared with the control group. D, E: Double immunostaining for HO-1 (green) and ED2 (red) showed that many HO-1-positive cells were ED2 macrophages. F: HO-1 mRNA levels in isolated glomeruli of statin-treated rats were up-regulated when comparing with those of the control rats. Data in $\mathbf{C}$ and $\mathbf{F}$ are mean $\pm \mathrm{SD} ; n=5$ in each time point in each group. 

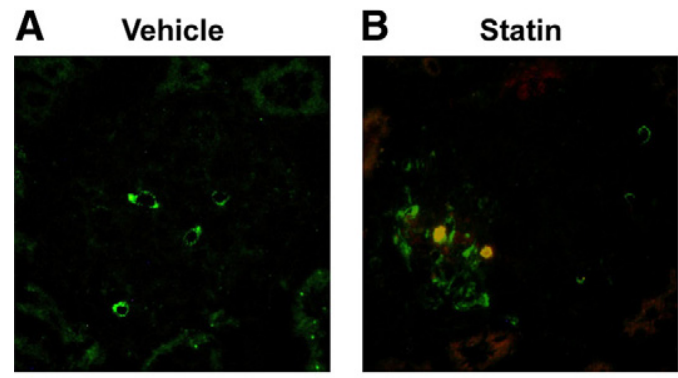

IL-10 (red) ED2 (green)

\section{Vehicle}
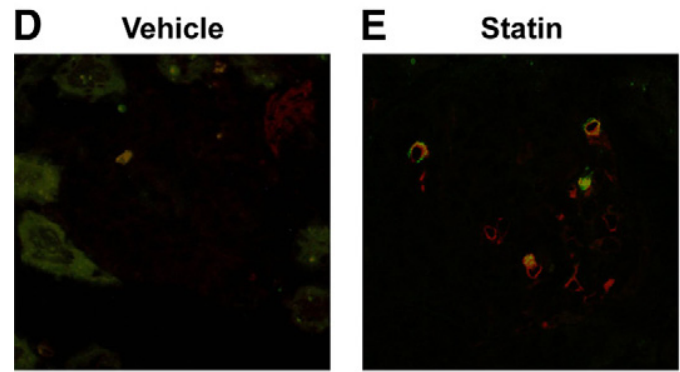

IL-10 (red) HO-1 (green)

\section{C mRNA of IL-10 \\ mRNA}

(normat

3

2

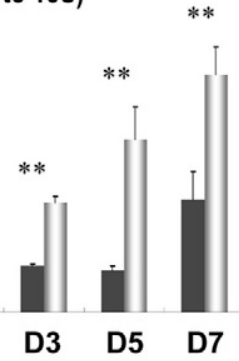

$* \mathrm{p}<0.05$

$* * \mathbf{p}<0.01$
Vehicle
Figure 10. Production of IL-10 in glomeruli of the control (vehicle) and statin groups. A, B Double immunostaining for IL-10 (red) and ED2 (green) showed both IL-10- and ED2-positive macrophages in glomeruli of the statin group. $\mathbf{C}$ IL-10 mRNA levels in isolated glomeruli of the control and statin groups showed that mRNA levels of IL-10 were up-regulated in the statin group as compared with those of the control group. D, E: Double immunostaining for IL-10 (red) and HO-1 (green) showed both IL-10- and HO-1-positive cells in glomeruli of statin-treated rats. Data in $\mathbf{C}$ are mean $\pm \mathrm{SD} ; n=5$ in each time point in each group.

\section{Discussion}

In the present study, anti-GBM GN in WKY rats was characterized by infiltration of mononuclear cells in the glomeruli from the early stage after disease induction, followed by the development of severe necrotizing and crescentic GN and profound proteinuria. Progression of GN was associated with accumulation of ED1+ and ED3+ macrophages in glomeruli and increased production of M1 macrophage-associated cytokines, IFN- $\gamma$, TNF- $\alpha$, and IL-12. On the other hand, atorvastatin (20 $\mathrm{mg} / \mathrm{kg} /$ day) reduced glomerular inflammation, necrotizing and crescent formation, resulting in reduction of pro-

\section{A CD11b macrophages B HO-1 expression}

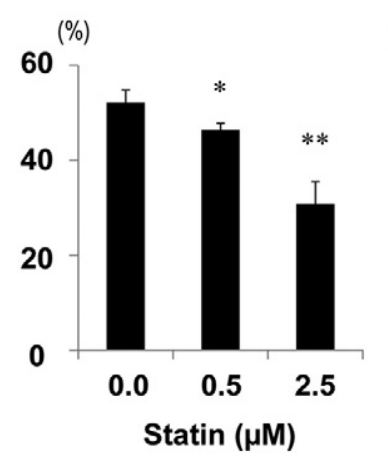

(Mean fluorescent intensity)

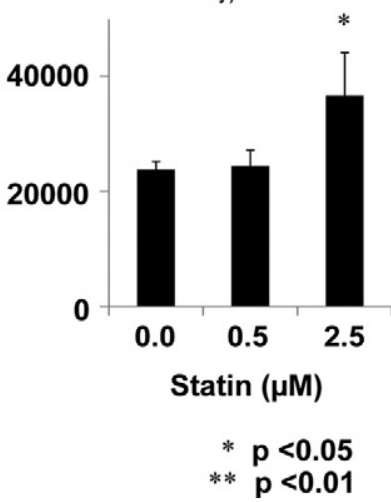

Figure 11. Inhibition of macrophage differentiation and increase of HO-1 expression by statin. U937 cells were cultured with $100 \mathrm{ng} / \mathrm{ml}$ of phorbol 12-myristate 13-acetate in the absence or presence of 0.5 and $2.5 \mu \mathrm{mol} / \mathrm{L}$ statin for 3 days. Cells were harvested and analyzed the percentage of CD11b-positive cells (A) and mean fluorescent intensity of HO-1 expression in CD11b-positive macrophages (B). Statin inhibited CD11b-positive macrophages in cultured U937 cells and enhanced HO-1 expression in CD11bpositive U937 cells. teinuria without alteration in serum lipid profile. The renoprotective effect of statin seems to be associated with inhibition of ED1 + macrophage infiltration through downregulation of glomerular MCP-1 expression, and reduction of ED3+ macrophages with down-regulation of M1related pro-inflammatory cytokines. Furthermore, statin augmented alternatively activated macrophages (M2) and $\mathrm{HO}-1+$ macrophages, together with up-regulation of anti-inflammatory cytokine, IL-10. These results indicate that statin does not only inhibit macrophage infiltration, but also induces M2 polarization through both the suppression of M1 development and augmentation of $\mathrm{M} 2$ and $\mathrm{HO}-1+$ macrophage development.

In the present study, severe crescentic GN was induced in WKY rats by administering a small dose of anti-GBM antibody. ${ }^{17}$ This model is characterized by massive accumulation of macrophages (ED1+ cells) in the glomeruli and by a high frequency of necrotizing glomerular lesions and crescent formation. Enhanced infiltration of macrophages in glomeruli by chemokines and adhesion molecules, such as MCP-1 and ICAM-1, is a crucial event for the initiation and subsequent progression of anti-GBM GN. ${ }^{36,37}$ In addition, depletion of macrophages results in marked suppression of necrotizing and crescentic glomerular lesions in this model, ${ }^{19,38}$ stressing the pivotal role for macrophages in glomerular injury and crescent formation.

Macrophage infiltration is a common feature in renal diseases, and their presence is synonymous with tissue damage and progressive renal failure. However, in macrophage-dependent inflammation, recent studies have also demonstrated the heterogeneity of activated macrophages and their ability to function not only to enhance but also suppress inflammation. ${ }^{13,20,22,30}$ LPS and proinflammatory stimuli such as IFN- $\gamma$ or TNF- $\alpha$, induce classically activated M1 macrophages, which produce pro- 


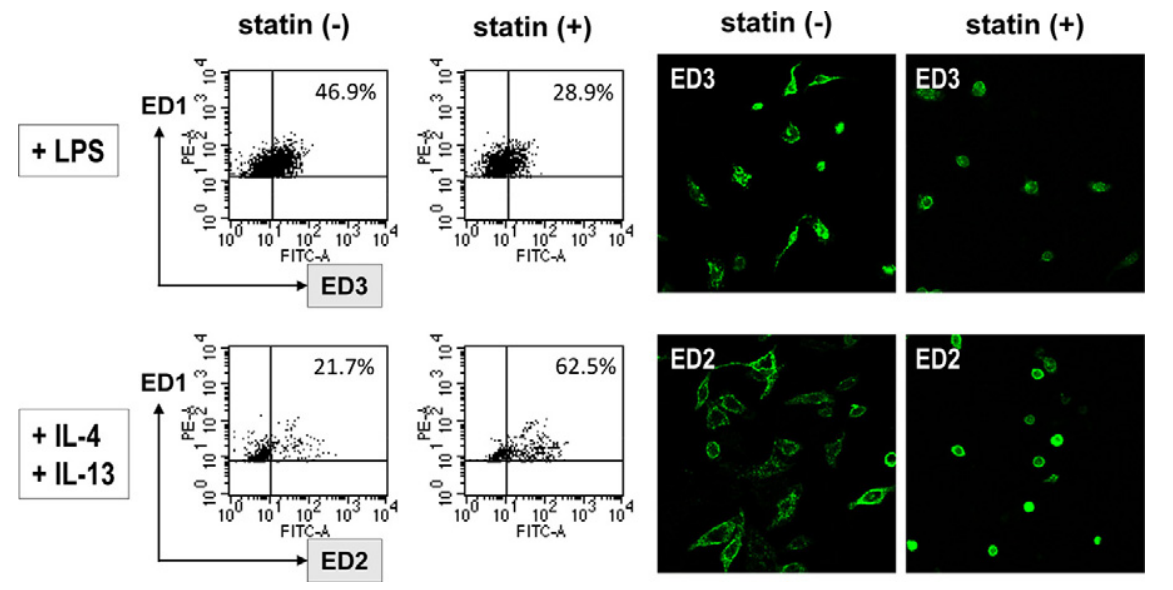

Figure 12. Effects of statin on M1 and M2 macrophage differentiation in vitro. Rat peritoneal macrophages were stimulated with LPS (M1 condition) or IL-4 and IL-13 (M2 condition) for 24 hours in the presence or in the absence of 2.5 $\mu \mathrm{mol} / \mathrm{L}$ statin. In the results of the flow cytometric analysis, cells were gated on ED1-positive macrophages, and percentage of ED3- or ED2positive cells in total ED1-positive macrophages was indicated. Statin inhibited the development of ED3-positive macrophages under LPS stimulation. On the other hand, statin augmented ED2-positive macrophages under IL-4 and IL-13 stimulation. In confocal scanning laser micrographs, the morphology of rat peritoneal macrophages was characterized by enlarged spindle, stellate, and polygonal shaped cells after LPS or IL-4 and IL-13 stimulation. However, statin inhibited these morphological changes even after LPS or IL-4 stimulation. inflammatory cytokines, IFN- $\gamma, \mathrm{TNF}-\alpha$, and IL-12. M1 macrophages have antimicrobial and cytotoxic properties, which underlie their role in host responses to infection. M1 macrophages are also involved in various macrophage-dependent tissue injuries and several autoimmune diseases, such as rheumatoid arthritis and inflammatory bowel disease. ${ }^{39,40}$ ED3 is sialoadhesin (CD169), which was originally identified as an erythrocyte receptor expressed by subsets of "activated" macrophages, and ED3+ cell accumulation has been described in chronic inflammatory lesions, including GN. ${ }^{31,32}$ In the present study, many ED3+ cells accumulated in glomeruli with up-regulation of pro-inflammatory cytokines, IFN- $\gamma$, TNF- $\alpha$, and IL-12 during the progression of anti-GBM GN. In addition, ED3+ macrophages expressed MCP-1, TNF- $\alpha$, and iNOS. In rat anti-GBM GN model, ED3+ macrophages seemed to have biological features similar to M1 macrophages.

On the other hand, alternatively activated M2 macrophages play an important role in immunoregulatory and immunosuppressive responses. ${ }^{30}$ The M2 macrophage activation pathway typically deactivates macrophages after exposure to Th2-type cytokines, such as IL-4 and IL-13. ${ }^{13,21,22,30}$ M2 macrophages express mannose receptor and secrete CCL17 and IL-10, but only little or no TNF- $\alpha$, IFN- $\gamma$, and IL-12. ${ }^{30,34}$ Unlike M1 macrophages, the M2 macrophages seem to help resolve inflammation through high endocytic clearance capacities and production of IL-10, TGF- $\beta$, and CCL17, in addition to reduction of pro-inflammatory cytokine secretion. In rats, ED2 + macrophages are considered equivalent to M2 macrophages, because rat ED2 is identified CD163, which is known to be expressed on M2 macrophages. ${ }^{22,29,30}$ In the present study, statin increased the number of ED2+ macrophages, although it decreased the total number of glomerular macrophages, and augmented M2 macrophage-associated chemokine and cytokine, CCL17 and IL-10. CCL17 is a chemokine known as thymus and activation regulated chemokine (TARC), which induces selective migration of lymphocytes, especially the Th2 phenotype. ${ }^{41}$ In addition, CCL17 has antiinflammatory effect also by inhibiting the immunological functions expressed by classically activated macrophages. ${ }^{30,34}$ In the present study, ED2+ macrophages in glomeruli in the statin group expressed CCL17. IL-10 has an important anti-inflammatory role with a wide-spectrum biological effect on dendritic cells, lymphocytes, and macrophages. ${ }^{42} \mathrm{IL}-10$ can inhibit macrophage activation, through the suppressing the production of pro-inflammatory mediators, including TNF- $\alpha$, IL-1 $\beta$, macrophage colony-stimulating factor, and reactive oxygen species. Thus, CCL17 and IL-10 produced by M2 macrophages might contribute to the inhibition of glomerular inflammation in this model. Recently, M2 macrophages have been further subdivided into $\mathrm{M} 2 \mathrm{a}, \mathrm{M} 2 \mathrm{~b}$, and $\mathrm{M} 2 \mathrm{c}$, or regulatory, wound-healing, and tumor-associated macrophages. ${ }^{21,43}$ Further study is important to examine the detailed characteristics and functions of macrophages after the statin treatment, as well as the anti-inflammatory functions.

In response to Th1- and Th2-type cytokines, macrophages express specialized and polarized functional properties, and differentiate into M1 or M2 macrophages. ${ }^{44}$ Th1- and Th2-type cytokines are secreted by mainly $\mathrm{CD}^{+}{ }^{+}$Th1 or Th2 cells. In the present study, only a few $\mathrm{CD}^{+}{ }^{+}$cells infiltrated the glomeruli $(0.2 \pm 0.4$ cells in the control group vs $0.3 \pm 0.5$ cells in the statin group per glomerular cross section on day 7 ). However, in the statin group, $\mathrm{CD}^{+}$cell-derived glomerular IL-4 was upregulated after statin administration. These results suggested that, although the number of $\mathrm{CD} 4^{+}$cells was very low and not significantly different between control and statin groups, a few of these $\mathrm{CD}^{+}$cells contributed to the suppression of glomerular inflammation through Th2type cytokines. On the other hand, similar to CD4 ${ }^{+}$Th1 cells, CD8+ cells play an important role in cellular cytotoxicity and macrophage activation through the production of Th1-type cytokines, including INF- $\gamma \cdot{ }^{45}$ Contrary, $\mathrm{ED} 3+$ macrophages may also promote T-cell immunity. ED3 (CD169) has been identified as a lymphocyte adhesion molecule, suggesting that ED3+ macrophages may contribute to the recruitment of lymphocytes in inflammatory sites. ${ }^{46}$ In addition, ED3+ macrophages have antigen presentation functions through the process exogenous antigens and stimulate MHC class I peptiderestricted CTL response by CD8+ cells. ${ }^{47}$ These findings suggest that the inflammatory injuries by CD8+ cells and macrophages enhance with a positive augmentation loop 
between ED3+ macrophages and CD8+ cells. In the present study, many CD8+ cells infiltrated the glomeruli in control group, but statin inhibited the infiltration of CD8+ cells into glomeruli $(5.6 \pm 0.5$ cells in the control group vs $4.0 \pm 0.5$ cells in the statin group per glomerular cross section on day $7, P<0.05$ ). Further studies are warranted to elucidate the effects of statin not only for macrophage activation but also for $T$ cell activation, as well as the alterations of immunomodulatory properties in this model. Indeed, several studies have demonstrated the effects of statin on endothelial cells, neutrophil, and T cell and B cell activation, as well as the alterations of immunomodulatory properties. ${ }^{48-51}$

In addition to $\mathrm{M} 1$ and $\mathrm{M} 2$ phenotypes of macrophages, our study showed the appearance of HO-1+ macrophages after administration of statin. $\mathrm{HO}-1$ plays a central role in heme metabolism, and also protects cells from injury evoked by various oxidative stresses. ${ }^{52,53} \mathrm{~A}$ recent study demonstrates that statins can induce $\mathrm{HO}-1$ in infiltrating macrophages both in vitro and in vivo, and that HO-1 + macrophages can act to limit local tissue destruction and support tissue repair by local delivery of $\mathrm{HO}-1$ in the ischemia-reperfusion acute kidney injury model..$^{23}$ Our study also indicated that statin induced $\mathrm{HO}-1+$ macrophages, which might act as anti-inflammatory macrophages by producing IL-10 in glomerular inflammation. Interestingly, CD163 (rat ED2) mediates hemoglobinheme uptake and activates $\mathrm{HO}-1$ in macrophages, which elicit IL-10 secretion. ${ }^{54}$ In addition, IL-10 induces HO-1 expression in murine macrophage cell line. ${ }^{52}$ These findings suggest that the anti-inflammatory process encompasses a positive augmentation loop between M2 macrophages, HO-1+ macrophages and IL-10.

In our in vitro study, the results of monocytic U937 cell line indicated that the statin affects monocytes directly and inhibited the macrophage differentiation as well as mediated HO-1+ anti-inflammatory macrophages. In rat peritoneal macrophages, statin inhibited the development of ED3+ macrophages. In addition, statin augmented ED2+ macrophages in M2-associaated cytokine environment. In our in vitro study, we cannot demonstrate the direct evidence of the phenotype transition of macrophages, M1 to M2, by statin. However, in our in vivo study, together with many ED2 single-positive and a few ED3 single-positive cells, both ED2+ and ED3+ cells were present in the glomeruli in the statin group. Both ED2+ and ED3+ cells may have both characterization of M1 and $\mathrm{M} 2$, and these results may suggest the possibility of transition of macrophages from M1 to M2 in local sites in vivo. Recent reports in vivo and vitro studies support our consideration that macrophages display remarkable plasticity and can change their physiology in response to environmental cues. ${ }^{43,55}$ It is very important to clarify the mechanism of statin-mediated augmentation of $\mathrm{M} 2$ with up-regulation of M2-related cytokines in inflammatory sites. Especially, it is mediated whether by direct effects of statin or indirect effects of statin through T cells or other immune-modulating properties.

In various experimental models of GN, statin exerts anti-inflammatory, and has several renoprotective, effects. ${ }^{1-3,23-25}$ Several mechanisms have been proposed to explain the renal beneficial effects of statins: 1) inhibition of mesangial proliferation, 2) inhibition of induction of TGF- $\beta$ and expansion of extracellular matrix, 3) inhibition of induction of MCP-1 and reduction of macrophage infiltration, 4) amelioration of inflammation and reduction of oxidative stress, 5) amelioration of podocyte damage, and 6) hemodynamic effects on endothelial function and vasodilatation. The present study indicated that, in addition to the reduction of glomerular macrophage infiltration through inhibition of MCP-1 production, statin inhibited M1 macrophage activation, as well as enhanced the development of $\mathrm{M} 2$ macrophages and $\mathrm{HO}-1+$ macrophages, and attenuated anti-GBM GN. To our knowledge, this is the first study to demonstrate the effects of statins on macrophage phenotype in an experimental $\mathrm{GN}$, including increased proportion of anti-inflammatory macrophages while decreasing the total number of infiltrating glomerular macrophages. Interventions designed to block M1 generation and/or enhance M2 polarity may be therapeutically feasible. The potential benefit of cellular therapies based on infusion of peripheral monocytes primed ex vivo by exposure to IL-4 and IL-13, to induce M2 macrophages, was reported in a mouse model of kidney disease. ${ }^{35}$ Similarly, the present study provides the rationale for the use of statin as a therapeutic agent to limit the progression of inflammatory diseases. The therapeutic application of statin in severe GN with extensive glomerular inflammation may be clinically useful, through not only inhibition of macrophage infiltration and $\mathrm{M} 1$ activation, but also through the increase in $\mathrm{M} 2$ macrophages.

\section{Acknowledgments}

We thank Ms. Mitue Kataoka, Kyoko Wakamatu, and Arimi Ishikawa, for excellent technical assistance.

\section{References}

1. Mason JC: Statins and their role in vascular protection. Clin Sci (Lond) 2003, 105:251-266

2. Mason JC: The statins-therapeutic diversity in renal disease? Curr Opin Nephrol Hypertens 2005, 14:17-24

3. Fried LF: Effects of HMG-CoA reductase inhibitors (statins) on progression of kidney disease. Kidney Int 2008, 74:571-576

4. Qian Q, Jutila MA, Van Rooijen N, Cutler JE: Elimination of mouse splenic macrophages correlates with increased susceptibility to experimental disseminated candidiasis. J Immunol 1994, 152:5000-5008

5. Nikolic-Paterson DJ, Atkins RC: The role of macrophages in glomerulonephritis. Nephrol Dial Transplant 2001, 16 Suppl 5:3-7

6. Chow F, Ozols E, Nikolic-Paterson DJ, Atkins RC, Tesch GH: Macrophages in mouse type 2 diabetic nephropathy: correlation with diabetic state and progressive renal injury. Kidney Int 2004, 65:116-128

7. Nguyen D, Ping F, Mu W, Hill P, Atkins RC, Chadban SJ: Macrophage accumulation in human progressive diabetic nephropathy. Nephrology (Carlton) 2006, 11:226-231

8. Ferrario F, Castiglione A, Colasanti G, Barbiano di Belgioioso G, Bertoli S, D'Amico G: The detection of monocytes in human glomerulonephritis. Kidney Int 1985, 28:513-519

9. Lan HY, Yang N, Nikolic-Paterson DJ, Yu XQ, Mu W, Isbel NM, Metz $\mathrm{CN}$, Bucala R, Atkins RC: Expression of macrophage migration inhibitory factor in human glomerulonephritis. Kidney Int 2000, 57:499-509

10. Ohashi R, Kitamura H, Yamanaka N: Peritubular capillary injury dur- 
ing the progression of experimental glomerulonephritis in rats. J Am Soc Nephrol 2000, 11:47-56

11. Tian S, Ding G, Jia R, Chu G: Tubulointerstitial macrophage accumulation is regulated by sequentially expressed osteopontin and macrophage colony-stimulating factor: implication for the role of atorvastatin. Mediators Inflamm 2006, 2006:12919

12. Ohashi R, Shimizu A, Masuda Y, Kitamura H, Ishizaki M, Sugisaki Y, Yamanaka N: Peritubular capillary regression during the progression of experimental obstructive nephropathy. J Am Soc Nephrol 2002 , 13:1795-1805

13. Kluth DC, Erwig LP, Rees AJ: Multiple facets of macrophages in renal injury. Kidney Int 2004, 66:542-557

14. Ferrario F, Rastaldi MP: Necrotizing-crescentic glomerulonephritis in ANCA-associated vasculitis: the role of monocytes. Nephrol Dial Transplant 1999, 14:1627-1631

15. Shimizu A, Kitamura H, Masuda Y, Ishizaki M, Sugisaki Y, Yamanaka $\mathrm{N}$ : Rare glomerular capillary regeneration and subsequent capillary regression with endothelial cell apoptosis in progressive glomerulonephritis. Am J Pathol 1997, 151:1231-1239

16. Shimizu A, Masuda $Y$, Kitamura $H$, Ishizaki M, Sugisaki $Y$, Yamanaka $\mathrm{N}$ : Apoptosis in progressive crescentic glomerulonephritis. Lab Invest 1996, 74:941-951

17. Shimizu A, Masuda $Y$, Mori T, Kitamura H, Ishizaki M, Sugisaki $Y$, Fukuda Y: Vascular endothelial growth factor165 resolves glomerular inflammation and accelerates glomerular capillary repair in rat antiglomerular basement membrane glomerulonephritis. J Am Soc Nephrol 2004, 15:2655-2665

18. Kawasaki K, Yaoita E, Yamamoto T, Kihara I: Depletion of CD8 positive cells in nephrotoxic serum nephritis of WKY rats. Kidney Int 1992, 41:1517-1526

19. Isome M, Fujinaka H, Adhikary LP, Kovalenko P, El-Shemi AG, Yoshida Y, Yaoita E, Takeishi T, Takeya M, Naito M, Suzuki H, Yamamoto $\mathrm{T}$ : Important role for macrophages in induction of crescentic antiGBM glomerulonephritis in WKY rats. Nephrol Dial Transplant 2004 19:2997-3004

20. Gordon S, Taylor PR: Monocyte and macrophage heterogeneity. Nat Rev Immunol 2005, 5:953-964

21. Ricardo SD, van Goor H, Eddy AA: Macrophage diversity in renal injury and repair. J Clin Invest 2008, 118:3522-3530

22. Mosser DM: The many faces of macrophage activation. J Leukoc Biol 2003, 73:209-212

23. Gueler F, Park JK, Rong S, Kirsch T, Lindschau C, Zheng W, Elger M, Fiebeler A, Fliser D, Luft FC, Haller H: Statins attenuate ischemiareperfusion injury by inducing heme oxygenase-1 in infiltrating macrophages. Am J Pathol 2007, 170:1192-1199

24. Yoshimura A, Inui K, Nemoto T, Uda S, Sugenoya Y, Watanabe S, Yokota N, Taira T, Iwasaki S, Ideura T: Simvastatin suppresses glomerular cell proliferation and macrophage infiltration in rats with mesangial proliferative nephritis. J Am Soc Nephrol 1998, 9:2027-2039

25. Usui H, Shikata K, Matsuda M, Okada S, Ogawa D, Yamashita T, Hida K, Satoh M, Wada J, Makino H: HMG-CoA reductase inhibitor ameliorates diabetic nephropathy by its pleiotropic effects in rats. Nephrol Dial Transplant 2003, 18:265-272

26. Campese VM, Park J: HMG-CoA reductase inhibitors and the kidney. Kidney Int 2007, 71:1215-1222

27. Zhang W, Liu M, Wu Y, Zhu P, Yin C, Gu M: Protective effects of atorvastatin on chronic allograft nephropathy in rats. J Surg Res 2007, 143:428-436

28. Lawman S, Mauri C, Jury EC, Cook HT, Ehrenstein MR: Atorvastatin inhibits autoreactive $B$ cell activation and delays lupus development in New Zealand black/white F1 mice. J Immunol 2004, 173:7641-7646

29. Mantovani A, Sozzani S, Locati M, Allavena P, Sica A: Macrophage polarization: tumor-associated macrophages as a paradigm for polarized M2 mononuclear phagocytes. Trends Immunol 2002, 23:549-555

30. Gordon S: Alternative activation of macrophages. Nat Rev Immunol 2003, 3:23-35

31. Ikezumi Y, Hurst LA, Masaki T, Atkins RC, Nikolic-Paterson DJ: Adoptive transfer studies demonstrate that macrophages can induce proteinuria and mesangial cell proliferation. Kidney Int 2003, 63:83-95

32. Ikezumi Y, Suzuki T, Hayafuji S, Okubo S, Nikolic-Paterson DJ, Kawachi H, Shimizu F, Uchiyama M: The sialoadhesin (CD169) expressing a macrophage subset in human proliferative glomerulonephritis. Nephrol Dial Transplant 2005, 20:2704-2713
33. Masuda Y, Shimizu A, Mori T, Ishiwata T, Kitamura H, Ohashi R, Ishizaki M, Asano G, Sugisaki Y, Yamanaka N: Vascular endothelial growth factor enhances glomerular capillary repair and accelerates resolution of experimentally induced glomerulonephritis. Am J Pathol 2001, 159:599-608

34. Katakura T, Miyazaki M, Kobayashi M, Herndon DN, Suzuki F: CCL17 and IL-10 as effectors that enable alternatively activated macrophages to inhibit the generation of classically activated macrophages. J Immunol 2004, 172:1407-1413

35. Wang Y, Wang YP, Zheng G, Lee VW, Ouyang L, Chang DH, Mahajan D, Coombs J, Wang YM, Alexander SI, Harris DC: Ex vivo programmed macrophages ameliorate experimental chronic inflammatory renal disease. Kidney Int 2007, 72:290-299

36. Kawasaki K, Yaoita E, Yamamoto T, Tamatani T, Miyasaka M, Kihara I: Antibodies against intercellular adhesion molecule-1 and lymphocyte function-associated antigen-1 prevent glomerular injury in rat experimental crescentic glomerulonephritis. J Immunol 1993, 150:1074-1083

37. Wada T, Yokoyama H, Furuichi K, Kobayashi KI, Harada K, Naruto M Su SB, Akiyama M, Mukaida N, Matsushima K: Intervention of crescentic glomerulonephritis by antibodies to monocyte chemotactic and activating factor (MCAF/MCP-1). FASEB J 1996, 10:1418-1425

38. Lan HY, Nikolic-Paterson DJ, Mu W, Atkins RC: Local macrophage proliferation in the pathogenesis of glomerular crescent formation in rat anti-glomerular basement membrane (GBM) glomerulonephritis. Clin Exp Immunol 1997, 110:233-240

39. Zhang $X$, Mosser DM: Macrophage activation by endogenous danger signals. J Pathol 2008, 214:161-178

40. Szekanecz Z, Koch AE: Macrophages and their products in rheumatoid arthritis. Curr Opin Rheumatol 2007, 19:289-295

41. Bonecchi R, Bianchi G, Bordignon PP, D'Ambrosio D, Lang R, Borsatti A, Sozzani S, Allavena P, Gray PA, Mantovani A, Sinigaglia F: Differential expression of chemokine receptors and chemotactic responsiveness of type 1 T helper cells (Th1s) and Th2s. J Exp Med 1998, 187:129-134

42. Howard M, O'Garra A: Biological properties of interleukin 10. Immunol Today 1992, 13:198-200

43. Mosser DM, Edwards JP: Exploring the full spectrum of macrophage activation. Nat Rev Immunol 2008, 8:958-969

44. Mantovani A, Sica A, Sozzani S, Allavena P, Vecchi A, Locati M: The chemokine system in diverse forms of macrophage activation and polarization. Trends Immunol 2004, 25:677-686

45. Martinez FO, Sica A, Mantovani A, Locati M: Macrophage activation and polarization. Front Biosci 2008, 13:453-461

46. van den Berg TK, Breve JJ, Damoiseaux JG, Dopp EA, Kelm S, Crocker PR, Dijkstra CD, Kraal G: Sialoadhesin on macrophages: its identification as a lymphocyte adhesion molecule. J Exp Med 1992, 176:647-655

47. Muerkoster S, Rocha M, Crocker PR, Schirrmacher V, Umansky V: Sialoadhesin-positive host macrophages play an essential role in graft-versus-leukemia reactivity in mice. Blood 1999, 93:4375-4386

48. Greenwood J, Steinman L, Zamvil SS: Statin therapy and autoimmune disease: from protein prenylation to immunomodulation. Nat Rev Immunol 2006, 6:358-370

49. Hakamada-Taguchi R, Uehara Y, Kuribayashi K, Numabe A, Saito K, Negoro H, Fujita T, Toyo-oka T, Kato T: Inhibition of hydroxymethylglutaryl-coenzyme a reductase reduces Th1 development and promotes Th2 development. Circ Res 2003, 93:948-956

50. Kwak B, Mulhaupt F, Myit S, Mach F: Statins as a newly recognized type of immunomodulator. Nat Med 2000, 6:1399-1402

51. Eller P, Eller K, Wolf AM, Reinstadler SJ, Tagwerker A, Patsch JR, Mayer G, Rosenkranz AR: Atorvastatin attenuates murine anti-glomerular basement membrane glomerulonephritis. Kidney Int 77:428-435

52. Lee TS, Chau LY: Heme oxygenase- 1 mediates the anti-inflammatory effect of interleukin-10 in mice. Nat Med 2002, 8:240-246

53. Nath KA: Heme oxygenase-1: a provenance for cytoprotective pathways in the kidney and other tissues. Kidney Int 2006, 70:432-443

54. Abraham NG, Drummond G: CD163-Mediated hemoglobin-heme uptake activates macrophage $\mathrm{HO}-1$, providing an antiinflammatory function. Circ Res 2006, 99:911-914

55. Grinberg S, Hasko G, Wu D, Leibovich SJ: Suppression of $\mathrm{PLC}\{$ beta\} 2 by endotoxin plays a role in the adenosine A2A receptormediated switch of macrophages from an inflammatory to an angiogenic phenotype. Am J Pathol 2009, 175:2439-2453 